TRANSACTIONS OF THE

AMERICAN MATHEMATICAL SOCIETY

Volume 317, Number 1, January 1990

\title{
A FIXED POINT THEOREM FOR WEAKLY CHAINABLE PLANE CONTINUA
}

\author{
PIOTR MINC
}

\begin{abstract}
In this paper the fixed point theorem is proven for every plane acyclic continuum $X$ with the property that every indecomposable continuum in the boundary of $X$ is contained in a weakly chainable subcontinuum of $X$.
\end{abstract}

\section{INTRODUCTION}

It is unknown whether an acyclic plane continuum has the fixed point property (see [20, p. 70]). A plane continuum is acyclic if it does not separate the plane. There are, however, partial results for large classes of plane continua. In particular H. Bell [1], K. Sieklucki [19] and S. Iliadis [11] proved that every acyclic plane continuum with no indecomposable continuum in its boundary has the fixed point property. In [8] C. Hagopian proved that each acyclic arcwise connected plane continuum does not contain an indecomposable continuum in its boundary, and therefore has the fixed point property. Another result of this type was obtained by O. Hamilton [10] who proved the fixed point property for chainable continua. A continuum is chainable provided that for every positive number $\varepsilon$ there is an open cover $G_{1}, G_{2}, \ldots, G_{n}$ such that the diameter of $G_{i}$ is less than $\varepsilon$ for $i=1,2, \ldots, n$ and $G_{i} \cap G_{j} \neq \varnothing$ if and only if $|i-j| \leq 1$ for $i, j=1,2, \ldots, n$. Note that every chainable continuum can be embedded in the plane (see [3]). The aim of this note is to give a common generalization of the theorems of Hagopian and Hamilton. An arc is of course a chainable continuum. It will be proven that arcwise connectedness in the fixed point theorem for acyclic plane continua can be replaced by the condition that every pair of points of the continuum is contained in a chainable subcontinuum. Moreover, instead of chainable continua we will use a larger class of weakly chainable continua.

1.1 Definition (see [16]). A finite sequence of sets $G_{1}, G_{2}, \ldots, G_{n}$ is said to be a weak chain provided that $G_{i} \cap G_{j} \neq \varnothing$ for $|i-j| \leq 1$, where $i, j=1,2, \ldots, n$. A weak chain $G=\left(G_{1}, G_{2}, \ldots, G_{n}\right)$ is said to be a refinement of a weak chain $G^{\prime}=\left(G_{1}^{\prime}, G_{2}^{\prime}, \ldots, G_{n^{\prime}}^{\prime}\right)$ provided that there is a function $\phi:\{1,2, \ldots, n\} \rightarrow$

Received by the editors June 14, 1988 .

1980 Mathematics Subject Classification (1985 Revision). Primary 54H25, 54F20.

Key words and phrases. Fixed point property, plane continuum, weakly chainable. 
$\left\{1,2, \ldots, n^{\prime}\right\}$ so that $G_{i} \subset G_{\phi(i)}^{\prime}$ for and $|\phi(i)-\phi(j)| \leq 1$ for $|i-j| \leq 1$, where $i, j=1,2, \ldots, n$. A continuum $Y$ is weakly chainable if there is an infinite sequence $G^{1}, G^{2}, \ldots$ of finite open covers of $Y$ such that each $G^{k}$ is a weak chain, each element of $G^{k}$ has diameter less than $1 / k$ and $G^{k+1}$ is a refinement of $G^{k}$ for $k=1,2, \ldots$. In [16] it is proven that a continuum is weakly chainable if and only if it is a continuous image of the pseudo arc (cf. [17 and 7]).

The following theorem is the main result of this paper.

1.2 Theorem. Let $X$ be acyclic plane continuum. If for every indecomposable continuum $Z$ in the boundary of $X$ there is a weakly chainable subcontinuum of $X$ containing $Z$, then $X$ has the fixed point property.

Note that it follows from [ 9 and 14] that if every two points of $Z$ are contained in a weakly chainable subcontinuum of $X$ then $Z$ is contained in a weakly chainable subcontinuum of $X$.

There are examples of acyclic continua without the fixed point property which are either locally connected (see [4]) or contractible (see [12 and 13]), so the assumption that $X$ is planar cannot be omitted. It would be interesting to learn how it is for tree-like continua. The answer to the question whether the tree-like continua without the fixed point property presented in [2 and 18] are weakly chainable seems to be negative. Note that the fixed point theorem for arcwise connected tree-like continua was proven by $\mathrm{K}$. Borsuk in [5].

\section{Simple CANALS}

In this section we will recall some methods introduced by K. Sieklucki in [19].

For any set $L$ homeomorphic to a subset of the real line denoted by $\dot{L}$ the set of points not lying in the interior of some arc contained in $L$.

Let $L_{1}$ and $L_{2}$ be two plane sets. A point $x \in L_{1} \cap L_{2}$ is said to be a point of local cut of $L_{1}$ and $L_{2}$ provide that there are a neighborhood $V_{1}$ of $x$ in $L_{1}$ and a neighborhood $V_{2}$ of $x$ in $L_{2}$ such that $V_{1} \cap V_{2}=(x)$, and for each sufficiently small neighborhood $U$ of $x$ in the plane $V_{1}$ meets two components of $U-V_{2}$.

For any set $X \subset R^{2}$ let $[X]$ be the union of $X$ and all bounded components of $R^{2}-X$.

For any continuum $Z \subset R^{2}$ and any set $D \subset R^{2}-[Z]$ homeomorphic to the real half line $[0, \infty)$, an arc $L$ is called a bridge from a point $t \in D$ to $Z$ if $L \cap D=\{t\}, t$ is a point of local cut of $L$ and $D, L-\dot{L} \subset R^{2}-Z$, and $\dot{L} \subset Z$.

For any continuum $Z \subset R^{2}$ a set $D \subset R^{2}-[Z]$ homeomorphic to the real half line $[0, \infty)$ is called a simple canal in $Z$ provided that for every $t \in D-\dot{D}$ there is a bridge $L(t)$ from $t$ to $Z$ such that $\lim _{t \rightarrow \infty} \operatorname{diam} L(t)=0 \quad(t \rightarrow \infty$ according to the parametrization of $D)$. We can assume that $L(t)$ is so chosen 
that

$$
\left[Z \cup L\left(t^{\prime}\right)\right] \subset[Z \cup L(t)] \text { for } t^{\prime} \in D \cap[Z \cup L(t)]
$$

By $\operatorname{Lim} D$ we will denote the set $\operatorname{cl} D-D$.

The following theorem was not explicitly stated in [19] but its proof follows readily that of Auxiliary Theorem in [19, p. 270].

2.1 Theorem (K. Sieklucki). Let $X$ be an acyclic plane continuum and let $f$ be a continuous map of $X$ into itself without a fixed point. Then there are:

an indecomposable continuum $Z$ contained in the boundary of $X$ in $R^{2}$, a map $f^{*}: R^{2} \rightarrow R^{2}$ which is an extension of $f$ restricted to $Z$,

a piecewise linear simple canal $D$ in $Z$ and

a connected set $K$ contained and closed in $R^{2}-[Z]$.

such that

(i) $\operatorname{Lim} D=Z$,

(ii) $K \cap L(t) \neq \varnothing$ for every $t \in D-\dot{D}$, and

(iii) for every $t \in D-\dot{D}$ there is $t^{\prime} \in D \cap[Z \cup L(t)]$ so that $f^{*}\left(K \cap\left[Z \cup L\left(t^{\prime}\right)\right]\right)$ $\subset[Z \cup L(t)]-[Z]$.

Now, suppose that Theorem 1.2 is false. There are $f, f^{*}, Z, D$ and $K$ satisfying Theorem 2.1. This supposition and the notation will be kept throughout the rest of the proof.

\section{Pseudocovering spaces}

Let $\alpha$ be a positive number so that

$$
d(f(x), x)>4 \alpha \text { for every } x \in X, \text { where } d \text { denotes the distance. }
$$

Using lines parallel to the coordinate axes divide the plane into a countable collection $\mathscr{B}$ of nonoverlapping squares each with the diameter $\alpha$. Denote by $\mathscr{V}$ the set of all vertices of these squares. Without loss of generality one can assume that the boundary of $X$ does not intersect $\mathscr{V}$.

3.2 Lemma. There is a weakly chainable continuum $Y \subset X-\mathscr{V}$ containing Z.

Proof. Let $Y_{1} \subset X$ be a weakly chainable continuum containing $Z$. Since $Y_{1} \cap \mathscr{V}$ is finite, it is sufficient to replace $Y_{1}$ by a continuum with the same properties but intersecting $\mathscr{V}$ at less points than $Y_{1}$. Let $v$ be a point of $Y_{1} \cap \mathscr{V}$. Since $\mathscr{V}$ does not intersect the boundary of $X, v$ is in the interior of $X$. Let $Q \subset X$ be a disc containing $v$ in the interior and containing no points of $\mathscr{V}$ in the boundary. Denote by $Y_{2}$ the continuum $\left(Y_{1} \cup Q\right)-\operatorname{Int}(Q)$. To compete the proof it is sufficient to prove that $Y_{2}$ is weakly chainable.

Let $g$ be a map from the pseudoarc $P$ onto $Y_{1}$. Since $P$ is one dimensional there is an extension of the map $g$ restricted to $g^{-1}(\operatorname{Bd}(Q))$ to a map $\bar{g}$ from $g^{-1}(Q)$ onto $\operatorname{Bd}(Q)$. (See [6, Theorem 1.9.3, p. 90].) Observe that the map 
of $P$ onto $Y_{2}$, which is equal to $g$ on $P-g^{-1}(Q)$ and to $\bar{g}$ on $g^{-1}(Q)$, is continuous. Hence $Y_{2}$ is weakly chainable.

Let $Y$ be a continuum satisfying Lemma 3.2. Denote by $\delta$ the distance from $Y$ to $\mathscr{V}$. For every $S \in \mathscr{B}$ let $A(S)$ be the closure of $B(S, \delta / 2)-B(\mathscr{V}, \delta / 2)$, where $B(C, \varepsilon)$ is the open ball around $C$ with the radius $\varepsilon$. Denote by $\mathscr{A}$ the collection consisting of all $A(S)$, where $S$ belongs to $\mathscr{B}$. Observe that

the diameter of any element of $\mathscr{A}$ is less than $2 \alpha$.

Consider a finite sequence $A_{1}, A_{2}, \ldots, A_{n}$ of elements of $\mathscr{A}$. We will model on this sequence a space $E$ to some extent resembling a covering space. Let $E^{\prime}=\bigcup_{j=1}^{n} A_{j} \times\{j\}$. Let $\sim$ be the minimal equality relation on $E^{\prime}$ satisfying the condition $(z, j) \sim(z, j+1)$ for every $j=1,2, \ldots, n-1$ and $z \in A_{j} \cap A_{j+1}$. Define $E$ as $E^{\prime} / \sim$. The space $E$ will be called a pseudocovering space of the sequence $A_{1}, A_{2}, \ldots, A_{n}$. The natural projection of $E$ into $R^{2}$ will be denoted by $p$. The image of the set $A_{j} \times\{j\}$ under the projection of $E^{\prime}$ onto $E$ will be denoted by $\widetilde{A}_{j}$.

Note that $E$ is compact and $p \mid \widetilde{A}_{j}$ is a homeomorphism of $\tilde{A}_{j}$ onto $A_{j}$.

3.4 Lemma. Let $g_{1}$ and $g_{2}$ be two continuous maps of a connected set I into $E$ so that $g_{1}(I)$ intersects each $\tilde{A}_{j}$ for $j=1,2, \ldots, n$. Then there is a point $t \in I$ so that $g_{1}(t)$ and $g_{2}(t)$ are in $\tilde{A}_{i}$ for some $i=1,2, \ldots, n$.

Proof. Observe that for every $j=1,2, \ldots, n$ the set $E-\tilde{A}_{j}$ is the union of two disjoint open sets $L_{j}=\bigcup_{i=1}^{j-1} \tilde{A}_{i}-\tilde{A}_{j}$ and $R_{j}=\bigcup_{i=j+1}^{n} \tilde{A}_{i}-\tilde{A}_{j}$. Consider the set $L=\left\{t \in I \mid\right.$ if, for some $j, g_{1}(t) \in \widetilde{A}_{j}$ then $\left.g_{2}(t) \in L_{j} \cup \widetilde{A}_{j}\right\}$. Observe that $L$ is a closed set. Suppose that the lemma is false. Then $L$ is a proper subset of $I$ and $L$ is also open in $I$. This is contradicts connectivity of $I$.

3.5 Lemma. There is a sequence $A_{1}, A_{2}, \ldots, A_{n}$ with the pseudocovering space $E$ that contains a continuum $\tilde{Y}$ so that $\rho(\tilde{Y})=Y$.

Proof. Since $Y$ is weakly chainable there is a sequence $G^{1}, G^{2}, \ldots$ of finite open covers of $Y$ satisfying Definition 1.1. Suppose that each weak chain $G^{k}$ is comprised of $G_{1}^{k}, G_{2}^{k}, \ldots, G_{n_{k}}^{k}$. By the definition there is a function $\phi_{k}:\left\{1,2, \ldots, n_{k+1}\right\} \rightarrow\left\{1,2, \ldots, n_{k}\right\}$ so that $G_{i}^{k+1} \subset G_{\phi_{k}(i)}^{k}$ for $i=$ $1,2, \ldots, n_{k+1}$, and $\left|\phi_{k}(i)-\phi_{k}(j)\right| \leq 1$ for $i, j=1,2, \ldots, n_{k+1}$ and $|i-j| \leq$ 1 . For every positive integer $k$ let $\psi_{k}$ denote a function of $\left\{1,2, \ldots, n_{k}\right\}$ into $\left\{1,2, \ldots, n_{1}\right\}$ so that $\psi_{k+1}=\psi_{k} \phi_{k}$ and $\psi_{1}$ is the identity on $\left\{1,2, \ldots, n_{1}\right\}$. Since the union of the interiors of elements of $\mathscr{A}$ contains $Y$, we can assume without loss of generality that there is a sequence $A_{1}, A_{2}, \ldots, A_{n_{1}}$ of elements of $\mathscr{A}$ so that $G_{i}^{1} \subset A_{i}$ for $i-1, \ldots, n_{1}$. Let $E$ be the pseudocovering space of $A_{1}, A_{2}, \ldots, A_{n_{1}}$. Denote by $C_{k}$ the closure of the set $\bigcup_{i=1}^{n_{k}}\left(\widetilde{A}_{\psi_{k}(i)} \cap p^{-1}\left(G_{i}^{k}\right)\right)$. Let $\tilde{Y}=\bigcap_{k=1}^{\infty} C_{k}$. Since $G_{i}^{k} \subset A_{\psi_{k}(i)}$, we have that $p(\tilde{Y})=Y$. Observe 
that the intersection of $\widetilde{A}_{\psi_{k}(i)} \cap p^{-1}\left(G_{i}^{k}\right)$ and $\widetilde{A}_{\psi_{k}(i+1)} \cap p^{-1}\left(G_{i+1}^{k}\right)$ is not empty for every positive integer $k$ and $i=1,2, \ldots, n_{k}-1$. Since the diameter of $\tilde{A}_{\psi_{k}(i+1)} \cap p^{-1}\left(G_{i+1}^{k}\right)$ is less than $1 / k, \tilde{Y}$ is connected and the proof of the lemma is complete.

3.6 Lemma. There is a sequence $A_{1}, A_{2}, \ldots, A_{n}$ with the pseudcovering space $E$ that contains a continuum $\widetilde{Z}$ so that $p(\tilde{Z})=Z$.

Proof. Let $A_{1}, A_{2}, \ldots, A_{n}, E$ and $\tilde{Y}$ be as in the preceding lemma. Since $Z \subset Y$ and $p(\tilde{Y})=Y$, there is a component $\tilde{Z}$ of $\tilde{Y} \cap p^{-1}(Z)$ so that $p(\tilde{Z})$ meets an internal composant of $Z$ (see [14]). Suppose that $Z-p(\widetilde{Z})$ is not empty. Then there is a neighborhood $V$ of $\widetilde{Z}$ in $\widetilde{Y}$ so that $Z-p(V)$ is not empty and the boundary of $V$ does not meet $p^{-1}(Z)$. There is a component $S$ of $V$ so that $p(S)$ meets an internal composant of $Z$. By [15, $\S 47$, III, Theorem 1] $S$ meets the boundary of $V$. So the continuum $p(S) \subset X$ meets an internal composant of $Z$ and is not contained in $Z$, which contradicts Theorem 2.1 of [14].

Throughout the rest of this paper $A_{1}, A_{2}, \ldots, A_{n}$ will denote the shortest sequence of elements of $\mathscr{A}$ with the pseudocovering space $E$ containing a continuum $\tilde{Z}$ so that $p(\widetilde{Z})=Z$.

Let dist denote the Hausdorff metric (see [15, §42, II])

3.7 Lemma. There is a positive number $\beta$ so that if $C \subset E$ is a continuum and $\operatorname{dist}(p(C), Z)<\beta$ then $C$ intersects each $\widetilde{A}_{i}$ for $i=1, \ldots, n$.

Proof. Suppose that the lemma is false. Then there is an index $i$ and there is a sequence of continua $C_{1}, C_{2}, \ldots$ contained in $E-\widetilde{A}_{i}$ so that the sequence $p\left(C_{1}\right), p\left(C_{2}\right), \ldots$ converges to $Z$ in the Hausdorff metric. Without loss of generality one can assume that the sequence $C_{1}, C_{2}, \ldots$ converges to a continuum $C$. Observe that $p(C)=Z$. But $C$ is contained in the pseudocovering space of either $A_{1}, A_{2}, \ldots, A_{i-1}$ or $A_{i+1}, \ldots, A_{n}$ which contradicts the choice of the sequence $A_{1}, A_{2}, \ldots, A_{n}$.

\section{LIFTING OF PATHS}

Let $B$ be a subset of the real line which is the union of a finite collection of closed intervals and let $g$ be a map of $B$ into the plane. Denote by $T_{g}$ the set of points $x \in g(B)$ for which $g^{-1}(x)$ is not a single point Let $t_{g}$ denote the set $g^{-1}\left(T_{g}\right)$. If $g$ is piecewise linear it will be called a $B$-path. In the case where $B$ is an interval a $B$-path will be called simply a path. Observe that if $g$ is a $B$-path then $t_{g}$ is finite.

We will say that a path $g$ mapping an interval $I$ into the plane can be lifted to $E$ if there is a piecewise linear map $h$ of an interval $J$ onto $I$ and there is a map $\tilde{h}$ of $J$ into $E$ so that $p \tilde{h}=g h$.

Let $g$ be a $B$-path and let $S$ be either a simple closed curve or an arc meeting $g(B)$ at a finite number of points. Denote by $W(g, S)$ the set of 
points of $S \cap g(B)$ which are local cuts of $S$ and $g(B)$. Let $w(g, S)$ be the number of points of $W(g, S)$.

4.1 Lemma. Let $B$ be the union of mutually disjoint intervals $I_{1}, I_{2}, \ldots, I_{k}$. Let $Q$ be a disc and let $g$ be a B-path so that $g(B) \subset Q$ and $g(\dot{B}) \subset \operatorname{Bd}(Q)$. Then the following two statements are true.

(i) If $S \subset Q$ is a simple closed curve missing $\operatorname{Bd}(Q) \cap g(B)$, intersecting $g(B)$ at a finite set and missing $T_{g}$, then the number $w(g, S)$ is even.

(ii) Let $L_{1}$ and $L_{2}$ be two arcs contained in $Q$. Suppose that $L_{1}$ and $L_{2}$ have common endpoints $u$ and $v$ which do not belong to $g(B)$. If $L_{1}$ and $L_{2}$ intersect $g(B)$ at a finite set and do not intersect $T_{g} \cup(\operatorname{Bd}(Q) \cap$ $g(B))$, then the number $w\left(g, L_{1}\right)-w\left(g, L_{2}\right)$ is even.

Proof of (i). It is enough to prove that the number $w\left(g \mid I_{i}, S\right)$ is even for $i=$ $1, \ldots, k$.

Let $Q^{*}$ be a disc containing $Q$ in its interior. There is a component of $Q^{*}-S$ containing $\operatorname{Bd}(Q)-S$. Let $Q_{1}$ be the closure of this component and let $Q_{2}$ be the closure of the other component of $Q^{*}-S$. Clearly $Q_{1} \cap Q_{2}=S$. Observe that for any $t \in I_{i}$ we have:

(a) if $g(t)$ is not a point of $W(g, S)$ then there is a neighborhood $V$ of $t$ in $I_{i}$ so that $g(V)$ is contained in either $Q_{1}$ or $Q_{2}$, and

(b) if $g(t)$ is a point of $W(g, S)$ then there two intervals $J_{1}$ and $J_{2}$ contained in $I_{i}$ and having $t$ as a common end point so that $g\left(J_{1}-\{t\}\right) \subset Q_{1}-S$ and $g\left(J_{2}-\{t\}\right) \subset Q_{2}-S$.

In other words $g\left(I_{i}\right)$ passes from one component of $Q^{*}-S$ to another at points of $W(g, S)$. Since both ends of $g\left(I_{i}\right)$ are in $Q_{1}-S$, then $w\left(g \mid I_{i}, S\right)$ is even.

Proof of (ii). Observe that since $L_{2} \cap g(B)$ is finite, $L_{1}$ can be replaced by an arc $L_{1}^{\prime}$ so that $w\left(g, L_{1}\right)=w\left(g, L_{1}^{\prime}\right)$ and $L_{1}^{\prime} \cap L_{2} \cap g(B)=\varnothing$. Thus without loss of generality we may assume that $L_{1} \cap L_{2} \cap g(B)=\varnothing$. By a similar argument we may assume that $L_{1} \cap L_{2}$ is finite.

For any two points $a$ and $b$ of $L_{i}$, let $\langle a, b\rangle_{i}$ denote the closed subarc of $L_{i}$ with the endpoints $a$ and $b$, where $i=1,2$.

Suppose that the lemma is false. Let $b$ be the first point of the arc $L_{1}$ (ordered from $u$ to $v$ ) so that $b \in L_{1} \cap L_{2}$ and $w\left(g,\langle u, b\rangle_{1}\right)-w\left(g,\langle u, b\rangle_{2}\right)$ is odd. Cleary $b \neq u$.

Let $a$ be the last point of the arc $\langle u, b\rangle_{1}$ (ordered from $u$ to $b$ ) which belongs to $\langle u, b\rangle_{2}-\{b\}$. Note that $e_{1}=w\left(g,\langle u, a\rangle_{1}\right)-w\left(g,\langle u, a\rangle_{2}\right)$ is even. Since the set $\langle a, b\rangle_{1} \cup\langle a, b\rangle_{2}$ is a simple closed curve, it follows from (i) that $e_{2}=w\left(g,\langle a, b\rangle_{1} \cup\langle a, b\rangle_{2}\right)$ is even. since the points $a$ and $b$ do not belong to $g(B)$ we have that $w\left(g,\langle a, b\rangle_{1} \cup\langle a, b\rangle_{2}\right)=w\left(g,\langle a, b\rangle_{1}\right)+w\left(g,\langle a, b\rangle_{2}\right)$ and $w\left(g,\langle u, b\rangle_{i}\right)=w\left(g,\langle u, a\rangle_{i}\right)+w\left(g,\langle a, b\rangle_{i}\right)$ for $i=1,2$. Observe that the number $w\left(g,\langle u, b\rangle_{1}\right)-w\left(g,\langle u, b\rangle_{2}\right)=e_{1}+e_{2}-2 w\left(g,\langle a, b\rangle_{2}\right)$ is even. This contradiction concludes the proof of the lemma. 
4.2 Lemma. There is a point $t$ in the simple canal $D$ so that every path $g$ mapping an interval $I$ into $[Z \cup L(t)]-[Z]$ can be lifted to $E$.

Proof. We will suppose that the lemma is false and prove the following claim.

Claim. Let $V$ be an open subset of the plane intersecting $Z$ and let $i$ be an integer between 1 and $n$. Then there is an open set $V^{\prime} \subset V$ so that $V^{\prime} \cap Z \neq \varnothing$ and $p^{-1}\left(V^{\prime}\right) \cap \widetilde{Z} \cap \tilde{A}_{i}=\varnothing$.

Proof of the claim. Observe that if the set $V \cap Z$ is not contained in $A_{i}$ then the claim is obvious. Since no open subset of an indecomposable continuum is contained in a simple closed curve, we can assume that there is a point $z \in V \cap Z$ belonging also to the interior of $A_{i}$.

Since we suppose that the lemma is false, we can assume that there is a path $g^{-}$mapping the interval $[-2,1]$ into $R^{2}-[Z]$ which cannot be lifted to $E$. Let $d_{1}$ be a point of $D$ so that $g^{-}([-2,-1]) \cap\left[Z \cup L\left(d_{1}\right)\right]=\varnothing$. Since $\operatorname{Lim} D=Z$ there is a point $d_{2} \in D$ so close to $Z$ that $L\left(d_{2}\right) \subset\left[Z \cup L\left(d_{1}\right)\right] \cap V$. Denote by $\tilde{d}_{2}$ the point in $\tilde{A}_{i}$ which is mapped by $p$ onto $d_{2}$. Let $d_{3} \in D$ be a point so that $L\left(d_{2}\right) \cap\left[Z \cup L\left(d_{3}\right)\right]=\varnothing$. We can assume that there is a path $g^{+}$mapping the interval $[1,2]$ into $\left.\left[Z \cup L\left(d_{3}\right)\right)\right]-[Z]$ which cannot be lifted to $E$.

Let $g$ be a path mapping $[-2,2]$ into $R^{2}-[Z]$ so that

$g$ restricted to $[-2,-1]$ is $g^{-}$,

$g$ restricted to $[1,2]$ is $g^{+}$,

$g^{-1}\left(d_{2}\right)=\{0\}$ and

$g([-2,2]) \cap\left(\left[Z \cup L\left(d_{1}\right)\right]-\left[Z \cup L\left(d_{3}\right)\right]\right) \subset D$.

Denote by $H$ the set of all maps $\tilde{h}$ of an interval $I$ into $E$ so that $\tilde{d}_{2} \in \tilde{h}(I)$ and there is a map $h$ of $I$ into $[-2,2]$ with $g h=p \tilde{h}$. Let $G$ be the union of the images of all elements of $H$. Since a path is a picewise linear map, the set $g^{-1}\left(p\left(G \cap \tilde{A}_{j}\right)\right)$ has a finite number of components for $j=1, \ldots, n$. Denote by $B_{j}$ the union of all nondegenerate components of $g^{-1}\left(p\left(G \cap \widetilde{A}_{j}\right)\right)$. Let $g_{j}$ denote the map $g$ restricted to $B_{j}$. Clearly $g_{j}$ is a $B_{j}$-path.

Now, we will prove that 2 and -2 do not belong to $\bigcup_{j=1}^{n} B_{j}$. Suppose, to the contrary, that $2 \in \bigcup_{j=1}^{n} B_{j}$. Then there is a map of $\tilde{h}$ an interval $I$ into $E$ and there is a map $h$ of $I$ into [-2,2] such that $2 \in h(I), g h=p \tilde{h}$ and $\tilde{d}_{2} \in \tilde{h}(I)$. Since $g^{-1}\left(d_{2}\right)=\{0\}, 0$ belongs to $h(I)$ and so does 1 . There is a component $J$ of $h^{-1}([1,2])$ which is mapped by $h$ onto [1,2]. Observe that $h$ and $\tilde{h}$ restricted to $J$ form a lifting of $g^{+}$to $E$. This contradicts the choice of $g^{+}$. The proof in the case of -2 is similar.

Next, we will prove that $g_{j}\left(\dot{B}_{j}\right) \subset \mathrm{Bd} A_{j}$. Let $q$ be a point from $\dot{B}_{j}$. There is a map $\tilde{h}$ of an interval $I=[a, b]$ into $E$ and there is a map $h$ of $I$ into $[-2,2]$ so that $\tilde{d}_{2} \in \tilde{h}(a), g h=p \tilde{h}, q=h(b)$ and $\tilde{h}(b) \in \tilde{A}_{j}$. Suppose that $g(q) \subset \operatorname{Int} A_{j}$. Then there is a subinterval $Q=[q, s]$ of $[-2,2]$ meeting $B_{j}$ at $q$ and mapped by $g$ into $A_{j}$. Let $J=[b, c]$ be an interval meeting 
$I$ at $b$. Define $h^{\prime}: I \cup J \rightarrow[-2,2]$ so that $h^{\prime}$ restricted to $I$ is $h$ and $h^{\prime}$ restricted to $J$ is a homeomorphism onto $Q$ (with $h^{\prime}(b)=q$ ). Clearly $\tilde{h}$ can be extended to $\tilde{h}^{\prime}: I \cup J \rightarrow E$ so that $g h^{\prime}=p \tilde{h}^{\prime}$. Since $\tilde{h}^{\prime}(J) \subset \widetilde{A}_{j}, s \in B_{j}$, which contradicts the choice of $q$.

For every $j=1, \ldots, n$ we will define a relation $R_{j}$ on pairs of points from $\tilde{A}_{j}-G$. Let $u$ and $v$ be points from $\tilde{A}_{j}-G$. We will say that $u$ and $v$ are in the relation $R_{j}$ if there is an (possibly degenerated) arc $L \subset A_{j}$ so that

(a) $p(u)$ and $p(v)$ are the endpoints of $L$,

(b) ${ }_{j} L \cap g_{j}\left(B_{j}\right)$ is finite,

(c) ${ }_{j} L \cap\left(T_{g_{j}} \cup\left(\mathrm{Bd} A_{j} \cap g_{j}\left(B_{j}\right)\right)\right)=\varnothing$ and

(d) ${ }_{j} w\left(g_{j}, L\right)$ is even.

It follows from Lemma 4.1 (ii) that $R_{j}$ is an equivalence relation and it decomposes $\tilde{A}_{j}-G$ into at most two classes. Moreover if there is an arc $L \subset A_{j}$ satisfying the conditions $(\mathrm{a}),(\mathrm{b})_{j},(\mathrm{c})_{j}$ but not $(\mathrm{d})_{j}$ then $u$ and $v$ belong to different classes of $R_{j}$.

Observe that the relations $R_{j}$ and $R_{j+1}$ coincide on $\widetilde{A}_{j} \cap \widetilde{A}_{j+1}-G$ for $j=1, \ldots, n-1$. In fact, for any two points $u$ and $v$ from $\widetilde{A}_{j} \cap \widetilde{A}_{j+1}-G$ we can find an arc $L \subset \operatorname{Int}\left(A_{j} \cap A_{j+1}\right) \cup\{p(u), p(v)\}$ satisfying (a), (b) $)_{j}$ and (c) $)_{j}$. Clearly, $L$ satisfies the conditions $(\mathrm{b})_{j+1}$ and $(\mathrm{c})_{j+1}$. Since $g_{j}\left(B_{j}\right) \cap \operatorname{Int} A_{j+1}=$ $g_{j+1}\left(B_{j+1}\right) \cup$ Int $A_{j}$, we have that $w\left(g_{j}, L\right)=w\left(g_{j+1}, L\right)$. It follows from Lemma 4.1 (ii) that $u$ and $v$ are in the relation $R_{j}$ if and only if they are in the relation $R_{j+1}$.

Since $\bigcup_{k=1}^{j} \widetilde{A}_{k} \cap \widetilde{A}_{j+1}=\widetilde{A}_{j} \cap \widetilde{A}_{j+1}$, the relations $R_{1}, R_{2}, \ldots, R_{n}$ can be successively extended to an equality relation $R$ defined on $E-G$.

Note that any two points from one component of $E-G$ are in the relation $R$ because they can be joined in by an arc. Denote by $u$ and $v$ two points of $\widetilde{A}_{i}$ which are mapped by $p$ onto the ends of the bridge $L\left(d_{2}\right)$. Since $L\left(d_{2}\right)$ intersects $g([-2,2])$ at $d_{2}$ which is a point of local cut, $u$ and $v$ are not in the relation $R_{i}$ and therefore not in the relation $R$. It follows that $u$ and $v$ are in different components of $E-G$. Since $G$ and $\widetilde{Z}$ are disjoint and $\widetilde{Z}$ is connected, one of the points $u$ and $v$ lies in a different component of $E-G$ than $\widetilde{Z}$. Let it be $v$. Let $\widetilde{V}$ be a neighborhood of $v$ in Int $\widetilde{A}_{i}$ contained entirely in the component of $v$ in $E-G$. The set $p(\widetilde{V})$ is open. Let $V^{\prime}$ be $p(\widetilde{V}) \cap V$. Since $L\left(d_{2}\right) \subset V$ and the point $p(v)$ being one of the ends of the bridge $L\left(d_{2}\right)$ belongs to $Z$, we have that $V^{\prime}$ intersects $Z$. The conditions $V^{\prime} \subset V$ and $p^{-1}\left(V^{\prime}\right) \cap \widetilde{Z} \cap \widetilde{A}_{i}=\varnothing$ follow readily from the construction of $V^{\prime}$. So the claim is true.

Using the claim one can construct a decreasing sequence $V_{1}, V_{2}, \ldots, V_{n}$ of open subsets of the plane so that $V_{i} \cap Z$ is not empty and $p^{-1}\left(V_{i}\right) \cap \tilde{Z} \cap \tilde{A}_{i}=\varnothing$ for $i=1,2, \ldots, n$. Now, since $p^{-1}\left(V_{n}\right) \cap \tilde{Z} \cap \widetilde{A}_{i}$ for $i=1,2, \ldots, n$, we have 
that no point of the set $V_{n} \cap Z$ is in $p(\widetilde{Z})$ contrary to the choice of $\widetilde{Z}$. This contradiction proves the lemma.

\section{Proof of TheOREM 1.2}

Let $t$ be a point of $D$ satisfying the conclusion of Lemma 4.2 and let $t^{\prime}$ be a point of $D$ satisfying the condition (iii) of 2.1. Since $d(f(x), x)>4 \alpha$ for $x \in X$ (see 3.1), there is $t_{0} \in D \cap\left[Z \cup L\left(t^{\prime}\right)\right]$ so that

$$
d\left(f^{*}(y), y\right)>3 \alpha \text { for } y \in\left[Z \cup L\left(t_{0}\right)\right] .
$$

Let $\beta$ be a number satisfying Lemma 3.7. By the conditions (i) and (ii) of 2.1 there is a continuum $F \subset K \cap\left[Z \cup L\left(t_{0}\right)\right]$ such that $\operatorname{dist}(F, Z)<\beta / 2$. There is a path $g$ mapping an interval $I$ into $\left[Z \cup L\left(t_{0}\right)\right]-[Z]$ so that $\operatorname{dist}(F, g(I))<\beta / 2$ and $f^{*}(g(I)) \subset[Z \cup L(t)]-[Z]$. By 4.2 there is a map $h$ of an interval $J$ onto $I$ and there is a map $\tilde{h}$ of $J$ into $E$ so that $p \tilde{h}=g h$. Since $p(\tilde{h}(J))=g(I)$ and $\operatorname{dist}(g(I), Z) \leq \operatorname{dist}(F, g(I))+\operatorname{dist}(F, Z)<\beta$, it follows from 3.7 that $\tilde{h}(J)$ intersects each $\tilde{A}_{i}$ for $i=1,2, \ldots, n$. There is a path $g_{1}$ mapping $J$ into $[Z \cup L(t)]-[Z]$ so that $d\left(g_{1}(q), f^{*} g h(q)\right)<\alpha$ for every $q \in J$. Using 5.1 we get

$$
d\left(g_{1}(q), g h(q)\right)>2 \alpha \quad \text { for every } q \in J .
$$

By 4.2 there is a map $h_{1}$ of an interval $J_{1}$ onto $J$ and there is a map $\tilde{h}_{1}$ of $J_{1}$ into $E$ so that $p \tilde{h}_{1}=g_{1} h_{1}$.

Consider the two maps $\tilde{h} h_{1}$ and $\tilde{h}_{1}$. Since $\tilde{h} h_{1}\left(J_{1}\right)$ intersects each $\tilde{A}_{i}$ for $i=1,2, \ldots, n$, it follows from 3.4 that there is a point $s \in J_{1}$ and there is an integer $j$ between 1 and $n$ so that $\tilde{h} h_{1}(s)$ and $\tilde{h}_{1}(s)$ are in $\tilde{A}_{j}$. Therefore the points $p \tilde{h} h_{1}(s)=g h h_{1}(s)$ and $p \tilde{h}_{1}(s)=g_{1} h_{1}(s)$ are in $A_{j}$. But this contradicts 5.2 because the diameter of $A_{j}$ is less than $2 \alpha$ (see 3.3). This contradiction completes the proof of Theorem 1.2.

Acknowledgments. The author is grateful to Professor J. Krasinkiewicz and Professor K. Sieklucki for the valuable discussions which influenced his understanding of the subject.

\section{REFERENCES}

1. H. Bell, On fixed point properties of plane continua, Trans. Amer. Math. Soc. 128 (1967), 539-548.

2. D. Bellamy, A tree-like continuum without the fixed point property, Houston J. Math. 6 (1979), $1-13$.

3. R. H. Bing, Snake-like continua, Duke Math. J. 18 (1951), 653-663.

4. K. Borsuk, Sur un continu acyclique qui se laisse transformer topologiquement en lui-meme sans point invariants, Fund. Math. 24 (1934), 51-58.

5. __ A theorem on fixed points, Bull. Acad. Sci. Polon. 2 (1954), 17-20.

6. R. Engelking, Dimension theory, PWN, Warsaw, 1978.

7. L. Fearnley, Characterizations of continuous images of pseudoarc, Trans. Amer. Math. Soc. 111 (1964), 380-399. 
8. C. Hagopian, A fixed point theorem for plane continua, Bull. Amer. Math. Soc. 77 (1971), $351-354$.

9. $\frac{1}{629}$, Another fixed point theorem for plane continua, Proc. Amer. Math. Soc. 3 (1972), 627629.

10. O. H. Hamilton, A fixed point theorem for pseudoarcs and certain other metric continua, Proc. Amer. Math. Soc. 2 (1951), 173-174.

11. S. Iliadis, Positions of continua in a plane and fixed points, Vestnik Moskov. Univ. 4 (1970), 66-70.

12. S. Kinoshita, On some contractible continua without fixed point property, Fund. Math. 40 (1953), 96-98.

13. R. Knill, Cones, products and fixed points, Fund. Math. 60 (1967), 35-46.

14. J. Krasinkiewicz, Concerning the boundaries of plane continua and the fixed point property, Bull. Acad. Sci. Polon. 21 (1973), 427-431.

15. K. Kuratowski, Topology, vol. 2, Academic Press, New York and Warsaw, 1968.

16. A. Lelek, On weakly chainable continua, Fund. Math. 51 (1962), 271-282.

17. J. Mioduszewski, A functional conception of snake-like continua, Fund. Math. 51 (1962), 179189.

18. L. G. Oversteegen and J. T. Rogers, Jr., An inverse limit description of an atriodic, tree-like continuum and an induced map without a fixed points, Houston J. Math. 6 (1980), 549-564.

19. K. Sieklucki, On a class of plane acyclic continua with the fixed point property, Fund. Math. 63 (1968), 257-278.

20. T. van der Walt, Fixed and almost fixed points, Mathematisch Centrum, Amsterdam, 1963.

Fat Mathematics, Auburn University, Auburn, Alabama 36849 